\title{
Pseudoquiste adrenal derecho
}

\author{
Right adrenal pseudocyst \\ Ilse Patatuchi-Rugerio, ${ }^{*}$ Aranxa Hernández-Rivas ${ }^{\ddagger}$
}

Palabras clave: Quiste adrenal, pseudoquiste, adrenalectomía, glándula suprarrenal, retroperitoneal.

Keywords: Adrenal cyst, pseudocyst, adrenalectomy, adrenal gland, retroperitoneal.

* Cirujano General.

‡ Enfermera General.

Hospital General de Zona No. 28, Instituto Mexicano del Seguro Social.

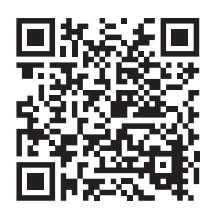

\section{RESUMEN}

El pseudoquiste adrenal es una entidad poco frecuente. Se han descrito alrededor de 600 casos desde su primer reporte. Pocos han sido informados en la literatura mexicana. Su diagnóstico y tratamiento pueden ser considerados un reto. A pesar de que existe controversia, la estrategia principal ha sido la misma en la última década. Presentamos el caso de un paciente con pseudoquiste suprarrenal derecho grande.

\section{ABSTRACT}

Adrenal pseudocyst is a rare entity. About 600 cases have been described since its first report. A few informed in Mexican Literature. Its diagnosis and treatment can be considered a challenge. Although there is controversy, main treatment strategy has been the same in the last decade. We present the case of a patient with a large right suprarenal pseudocyst.

\section{INTRODUCCIÓN}

$\mathrm{L}$ as lesiones adrenales fueron descritas por primera vez por Greiselius en 1670, un médico vienés. ${ }^{1}$ Se identifica a cualquier edad, en especial entre 30 y 60 años de edad. Más común en mujeres, ${ }^{2,3}$ pueden ser asintomáticos o presentarse con dolor, síntomas gastrointestinales y una masa palpable. ${ }^{4}$ Se han detectado en 0.06 a $0.18 \%$ de las autopsias. 5,6 Y son identificados en $4 \%$ de los escaneos por tomografía. $^{7}$

Acorde a la clasificación de Levison se dividen en cuatro categorías: quistes endoteliales (45\%), quistes epiteliales (9\%), pseudoquistes (39\%) y quistes parasitarios (7\%).6,8-11 Pueden medir desde milímetros hasta $50 \mathrm{~cm}$ de diámetro. ${ }^{12}$ Son unilaterales, pero se pueden presentar de forma bilateral (8-15\%).,12

Dentro de los factores predisponentes se encuentran trauma abdominal, hipoxia neonatal, diátesis hemorrágica, uso de anticoagulantes y aneurismas. ${ }^{10}$ La posible etiología del pseudoquiste adrenal incluye: malformación y hemorragia de las venas adrenales a la glándula adrenal por trauma, cirugía o coagulopatía. 3,9

\section{PRESENTACIÓN DEL CASO}

Paciente masculino de 42 años sin antecedentes de importancia acude a la consulta externa de cirugía general. Inicia en marzo de 2018 con dolor abdominal cólico en hipocondrio derecho, leve, irradiado a fosa renal derecha, intermitente y sin síntomas agregados.

Ultrasonido de marzo reporta hígado de tamaño, forma y situación normales con borde inferior derecho irregular, de ecogenicidad heterogénea por presencia de una masa anecoica de forma redondeada y con bordes bien delimitados, paredes delgadas; sugestivo de quiste simple (Figuras 1 y 2).

Biometría hemática, pruebas de función hepática, renales, TP y TPT de mayo de 2018 normales. No se realiza cortisol, aldosterona, catecolaminas urinarias ni metanefrinas. Tomografía de mayo reporta en glándula suprarrenal derecha imagen hipodensa con diámetros máximos de 101 mm, calcificaciones en su in-

Citar como: Patatuchi-Rugerio I, Hernández-Rivas A. Pseudoquiste adrenal derecho. Cir Gen. 2020; 42 (3): 215-218. https://dx.doi.org/10.35366/99962 
terior con efecto ocupativo y compresivo sobre las estructuras vecinas (Figuras 3 a 6). Completándose su valoración preoperatoria por medicina interna, se programa abordaje quirúrgico anterior abierto y bajo profilaxis antibiótica con ceftriaxona $2 \mathrm{~g}$ en dosis única, se realiza incisión subcostal derecha, destechamiento con electrocauterio de quiste suprarrenal derecho de $10 \times$ $9 \mathrm{~cm}$, con adherencias al hígado, contenido de aproximadamente $400 \mathrm{ml}$ de líquido hialino y calcificaciones de alrededor de $20 \times 40 \mathrm{~mm}$ en su interior. Se coloca drenaje abierto, penrose de $1 / 2$ " $(19 \mathrm{~mm})$ en espacio subfrénico ante la sospecha de hemorragia postoperatoria.

Reporte histopatológico de pseudoquiste adrenal asociado a hemorragia antigua, fibrosis y calcificación distrófica extensa. El paciente es egresado al tercer día de hospitalización con seguimiento por la consulta, hasta los cuatro meses del postoperatorio con ultrasonido hepático y renal derecho de control sin presentar complicaciones (Figura 7).

\section{DISCUSIÓN}

A diferencia de los quistes verdaderos los pseudoquistes adrenales no tienen un revestimiento celular, se componen de tejido fibrótico, algunas veces con calcificaciones. ${ }^{8,9}$

La mayoría son no funcionales y benignos. El estado funcional de los mismos puede causar síntomas de insuficiencia suprarrenal. El riesgo

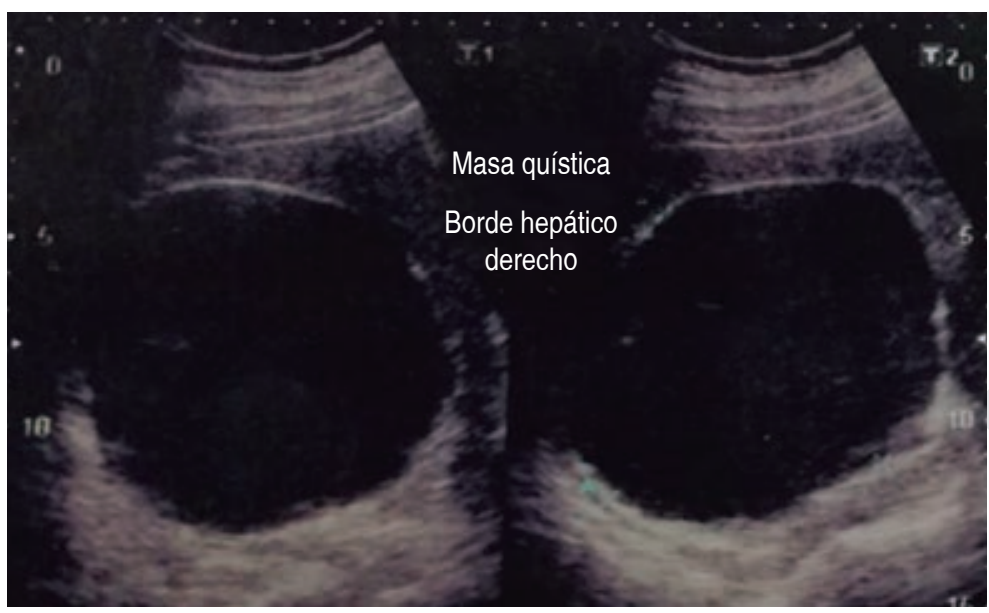

Figura 1: Ultrasonido hepático con imagen anecoica redondeada y de paredes delgadas bien delimitadas.

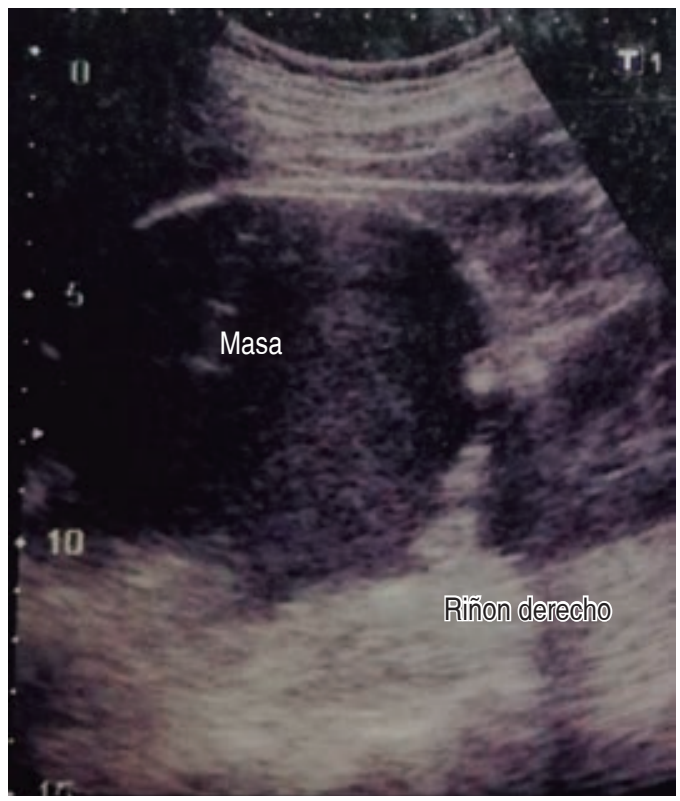

Figura 2: Ultrasonido con imagen de quiste simple, borde hepático inferior derecho.

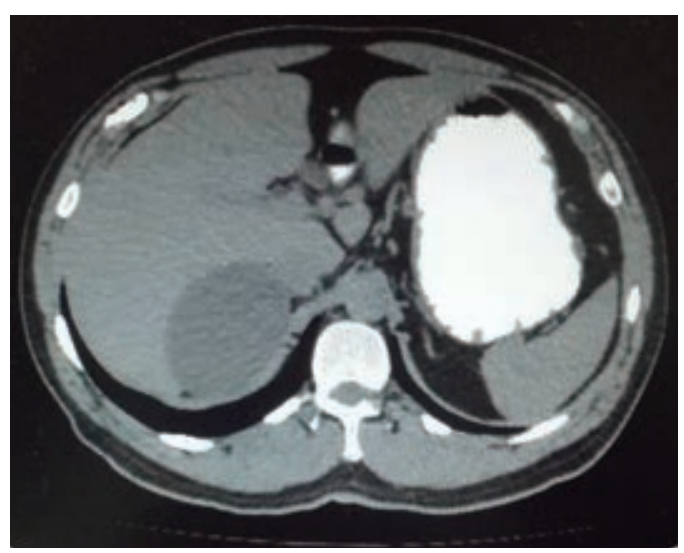

Figura 3: Imagen hipodensa con efecto ocupativo a glándula hepática.

de malignidad es de $7 \% .{ }^{11}$ El reconocimiento y diagnóstico de un carcinoma adrenal primario en estadio temprano es particularmente importante, por lo que una resección completa ofrece la única oportunidad de supervivencia del paciente. ${ }^{9,10}$ Las lesiones malignas adrenales más frecuentes son metástasis (95\%), feocromocitoma maligno (3\%) y carcinoma suprarrenal $(2 \%) .{ }^{12}$

Los estudios iniciales deben comprender biometría hemática, pruebas de función he- 
pática y renal, cortisol, aldosterona, calcio, catecolaminas urinarias y metanefrinas. ${ }^{3} \mathrm{El}$ ultrasonido es por lo regular el primer estudio de imagen empleado en la evaluación de una masa adrenal por su bajo costo y sin exposición a la radiación. Sin embargo, su sensibilidad varía de 66.7 a $90 \%$. En tomografía la sensibilidad es de $85-95 \%$ y especificidad de 95 a 100\%. La IRM tiene como limitante, a diferencia de la tomografía, baja sensibilidad para detectar calcificaciones. ${ }^{10} \mathrm{El}$ diagnóstico diferencial de un pseudoquiste adrenal esencialmente incluye todas las lesiones ocupantes de espacio del abdomen superior: quistes hepáticos, esplénicos y renales así como quistes mesentéricos o retroperitoneales y tumores sólidos adrenales. ${ }^{10}$ Debe tomarse en cuenta que un diagnóstico preoperatorio de un pseu-

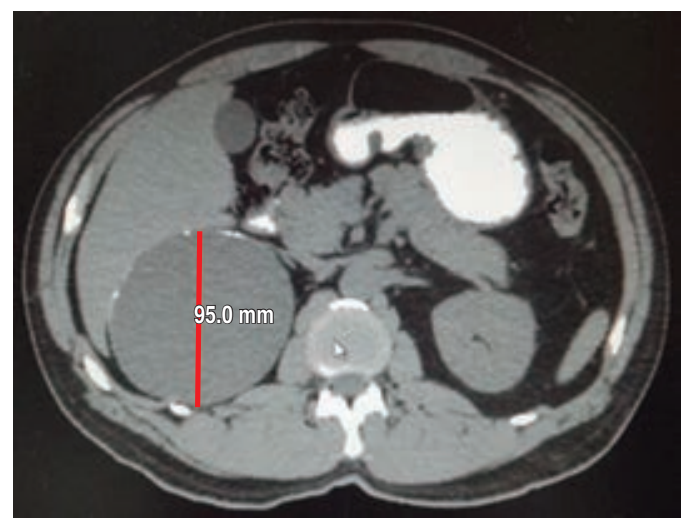

Figura 4: Imagen quística de 95 mm en su eje anteroposterior.

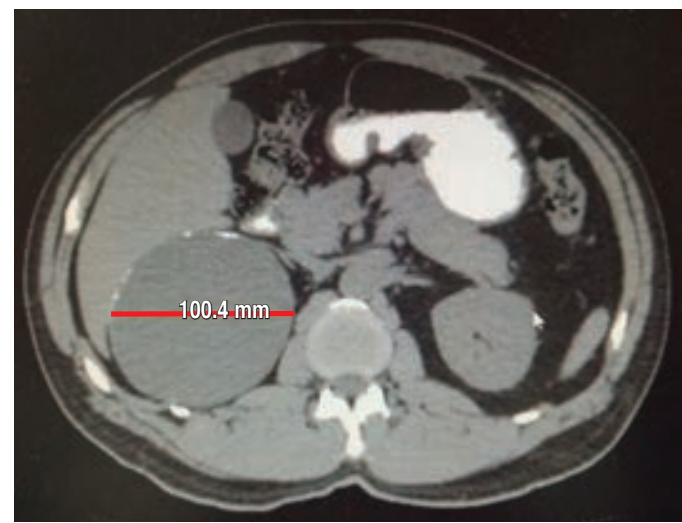

Figura 5: Imagen quística con calcificaciones, diámetro máximo de $100.4 \mathrm{~mm}$.

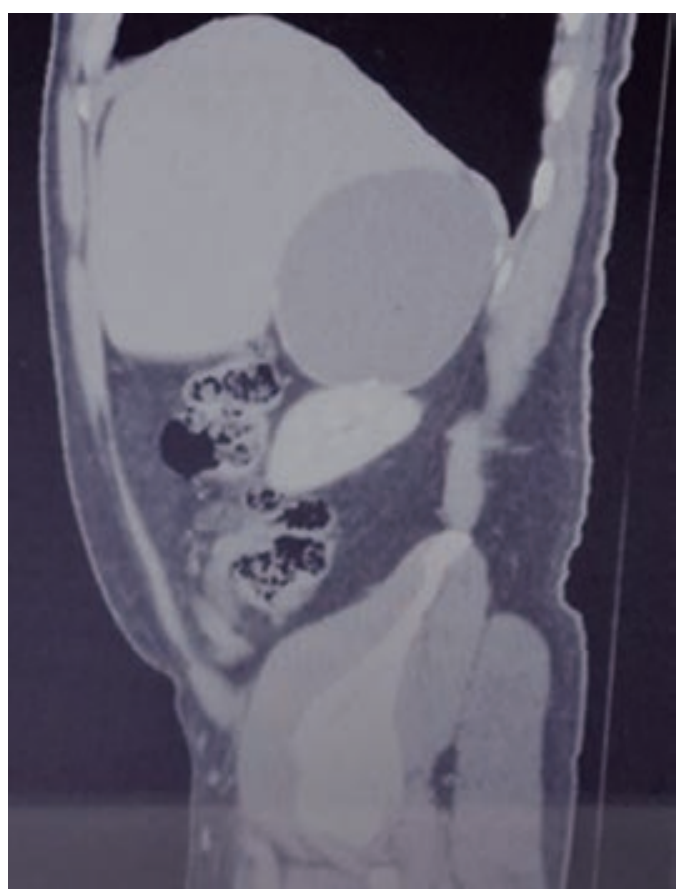

Figura 6: Imagen quistica grande con efecto ocupativo en estructuras vecinas.

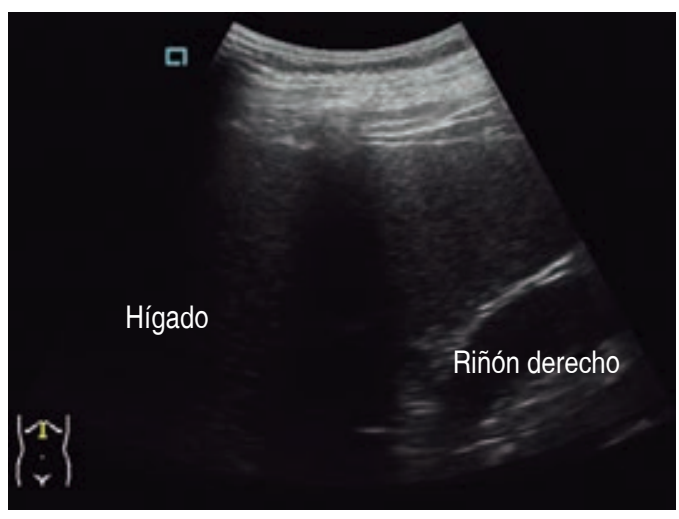

Figura 7: Ultrasonido hepático a los cuatro meses del posoperatorio, parénquima con ecogenicidad homogénea sin evidencia de lesiones focales.

doquiste grande puede ser en realidad muy difícil debido tanto a sus límites indistintos con los órganos circundantes como a la presencia de adherencias hacia los mismos. ${ }^{10}$

Para el tratamiento en quistes menores de $4 \mathrm{~cm}$ se aconseja repetir la tomografía a los tres meses, con un periodo de seguimiento de al menos 18 meses. ${ }^{7,10}$ La escisión quirúrgica se 
recomienda en quistes sintomáticos mayores de $5 \mathrm{~cm}$ de diámetro por el riesgo de malignidad y en pseudoquistes funcionales. El abordaje incluye: laparotomía, abordaje retroperitoneal abierto y laparoscópico. ${ }^{5}$ Otros procedimientos descritos son el destechamiento del quiste abierto o laparoscópico y la aspiración con una aguja vía percutánea. ${ }^{10} \mathrm{El}$ diagnóstico final será provisto por el examen de patología después del procedimiento quirúrgico.

\section{CONCLUSIÓN}

Existen varias maneras de tratar este tipo de quistes, dependiendo de las características de las lesiones, la experiencia del cirujano y los recursos locales. ${ }^{10}$

\section{REFERENCIAS}

1. Davenport M, Pollard K, Smith SE, MacMahon MJ. Adrenal cysts- report, review and classification. Postgrad Med J. 1988; 64: 71-73.

2. Chue KM, Goh G, Chiew A. Right adrenal gland pseudocyst masquerading as a large symptomatic hepatic cyst: single incision laparoscopic (SILS) resection an review of current literature. Ann Hepatobiliary Pancreat Surg. 2018; 22: 75-78.

3. Ujam AB, Peters CJ, Tadrous PJ, Webster JJ, Steer K, Martinez IA. Adrenal pseudocyst: diagnosis and laparoscopic management- a case report. Int J Surg Case Rep. 2011; 2: 306-308.

4. Cavallaro G, Crocetti D, Paliotta A, De Gori A, Rita TM, Letizia C, et al. Cystic adrenal lesions: clinical and surgical management. The experience of a referral centre. Int J Surg. 2015; 23-26.

5. Olaoye I, Dapo M, Abiodun E. A giant adrenal cyst with an uncertain preoperative diagnosis causing a dilemma in management. Clin Case Rep. 2018; 6: 1074-1076.

6. Lyu X, Liu L, Yang L, Gao L, Wei Q. Surgical management of cysts: a single-institution experience. Int Braz J Urol. 2014; 40: 656-665.
7. Ahmadreza S, Taha A, Marzieh L, Ali M. Gyany adrenal pseudocyst. Archieves of Iranian Medicine. 2013; 16: 741-742.

8. Carsote M, Ghemigian A, Terzea D, Gheorghisan GA, Valea A. Cystic adrenal lesions: focus on pediatric population (a review). Clujul Medica. 2017; 90: 5-12. Available in: https://www.ncbi.nlm.nih.gov/pmc/ articles/PMC5305088/pdf/cm-90-5.pdf.

9. Isono M, Ito K, Seguchi K, Kimura T, Tachi K, Kono T. A case of hemorragic adrenal pseudocyst mimicking solid tumor. Am J Case Rep. 2017; 18: 1034-1038. Available in: https://www.ncbi.nlm.nih.gov/pmc/ articles/PMC5627864/pdf/amjcaserep-18-1034.pdf.

10. Paramythiotis D, Bangeas P, Karakatsanis A, Goulas P, Nikolaou I, Rafailidis V, et al. Surgical management of a giant adrenal pseudocyst: a case report and review of the literature in the last decade. Case reports in surgery. 2018; 1-6. Available in: https://www.hindawi. com/journals/cris/2018/8473231/.

11. Kodama K, et al. Laparoscopic management of a complex adrenal cyst. Case Reports in Urology. 2015; 1-4. Available in: https://www.hindawi.com/journals/ criu/2015/234592/.

12. Cabané TP, Gac MP, Mariño BJ, Ibacache AD, Ledezma SA, Morales HC. Quiste suprarenal primario. Rev Chilena de Cirugía. 2011; 63: 617-622.

Consideraciones y responsabilidad ética: Privacidad de los datos. De acuerdo a los protocolos establecidos en el centro de trabajo de los autores, éstos declaran que han seguido los protocolos sobre la privacidad de datos de pacientes preservando su anonimato. El consentimiento informado del paciente referido en el artículo se encuentra en poder del autor.

Financiamiento: No se recibió apoyo financiero para la realización de este trabajo.

Conflicto de intereses: Los autores declaran que no existe ningún conflicto de intereses en la realización del trabajo.

Correspondencia:

Ilse Patatuchi-Rugerio

E-mail: ilselacr@hotmail.com 